\title{
Acknowledgement of manuscript reviewers 2014
}

Mohammad Abdollahi, Mohammad Ali Faramarzi, Alireza Foroumadi, Kambiz Gilani, Hossein Khalili, Mahnaz Khanavi, Ali Akbar Moghadamnia, Shekoufeh Nikfar, Ali Nokhodchi, Roja Rahimi, Mohammad Reza Rouini and Alireza Vatanara

\section{Contributing reviewers}

The editors of DARU Journal of Pharmaceutical Sciences would like to thank all our reviewers who have contributed to the journal in Volume 22 (2014).

\author{
Akbar Abdollahiasl \\ Iran \\ Farid Abedin Dorkoosh \\ Iran
}

Bahareh Abtahi Naeini

Iran

Reza Ahmadkhaniha

Iran

Sayed Mortaza Alavi Shoushtari

Iran

Alireza Aliabadi

Iran

Majed Al-Shaeri

Saudi Arabia

Amir Amani

Iran

Massoud Amanlou

Iran

Mohsen Amin

Iran

Ghazal Ansari

Iran

Amir Ansaripour

Netherlands

Kamaran Aryana

Iran

\author{
Ali Asadipour \\ Iran \\ Manouchehr Ashrafpour \\ Iran
}

Abbas Azadmehr

Iran

Homa Azizian

Iran

Mohammad Babaeian

Iran

Zaheer Babar

New Zealand

Haji Bahadar

Pakistan

Pejman Bakhtiari

United States of America

Behzad Baradaran

Iran

Ayse Nursen Basaran

Turkey

Joaquim Bosch

Spain

Jeffrey Brent

United States of America

Abdol Majid Cheraghali

Iran
Angela Dago

Spain

Simin Dashti-Khavidaki

Iran

Ilker Dastan

Turkey

Siavoush Dastmalchi

Iran

Luiza Dias

Brazil

Bharat Dixit

India

Reza Dowlatabadi

Iran

Najmeh Edraki

Iran

Saeed Emami

Iran

Mohammad Ali Faramarzi

Iran

Shadi Farsaei

Iran

Gholamhossein Farzandi

Iran

Loghman Firoozpour

Iran

* Correspondence: Mohammad@Sina.TUMS.Ac.Ir

DARU Journal of Pharmaceutical Sciences, Tehran, Iran 


Alireza Foroumadi
Iran
Akbar Fotouhi
Iran
Padideh Ghaeli
Iran
Alireza Ghaffarieh
United States of America
Doaa Ghareeb
Egypt
Younes Ghasemi
Iran
Kambiz Gilani
Iran

Mohammad Hadi Goharbari Iran

Gianpaolo Guzzi

Italy

Farzin Hadizadeh

Iran

Homa Hajimehdipoor

Iran

Javad Hamedi

Iran

Mehrdad Hamidi

Iran

Hamed Hamishehkar

Iran

Hadi Hamishehkar

Iran

Shirin Hasani-Ranjbar

Iran

Amir Hashemi

Iran

Atieh Hashemi

Iran

Mohammad Bagher Hashemi Iran

Salman Hassanzadeh

Sweden

A. Wallace Hayes

United States of America

Marjan Heidary

Iran

\begin{tabular}{|c|c|}
\hline $\begin{array}{l}\text { Bahram Hemmateenejad } \\
\text { Iran }\end{array}$ & $\begin{array}{l}\text { Jayashri Kulkarni } \\
\text { Australia }\end{array}$ \\
\hline $\begin{array}{l}\text { Narjes Hendouei } \\
\text { Iran }\end{array}$ & $\begin{array}{l}\text { Pravir Kumar } \\
\text { India }\end{array}$ \\
\hline $\begin{array}{l}\text { Farshad Homayouni Moghadam } \\
\text { Iran }\end{array}$ & $\begin{array}{l}\text { Caterina La Porta } \\
\text { Italy }\end{array}$ \\
\hline $\begin{array}{l}\text { Seyed Jalal Hosseinimehr } \\
\text { Iran }\end{array}$ & $\begin{array}{l}\text { Mohammad Mahdavi } \\
\text { Iran }\end{array}$ \\
\hline $\begin{array}{l}\text { Narges Hosseinmardi } \\
\text { Iran }\end{array}$ & $\begin{array}{l}\text { Soleiman Mahjoub } \\
\text { Iran }\end{array}$ \\
\hline $\begin{array}{l}\text { Hossein Hosseinzadeh } \\
\text { Iran }\end{array}$ & $\begin{array}{l}\text { Ghorban Maliji } \\
\text { Iran }\end{array}$ \\
\hline $\begin{array}{l}\text { Takashi Ikejima } \\
\text { China }\end{array}$ & $\begin{array}{l}\text { Azadeh Manayi } \\
\text { Iran }\end{array}$ \\
\hline $\begin{array}{l}\text { Mona Jaberidoost } \\
\text { Iran }\end{array}$ & $\begin{array}{l}\text { William Manzanares } \\
\text { Uruguay }\end{array}$ \\
\hline $\begin{array}{l}\text { Samineh Jafari } \\
\text { Iran }\end{array}$ & $\begin{array}{l}\text { Faheem Maqbool } \\
\text { Pakistan }\end{array}$ \\
\hline $\begin{array}{l}\text { Fakhreddin Jamali } \\
\text { Canada }\end{array}$ & $\begin{array}{l}\text { Ali Akbar Moghadamnia } \\
\text { Iran }\end{array}$ \\
\hline $\begin{array}{l}\text { Gholamreza Karimi } \\
\text { Iran }\end{array}$ & $\begin{array}{l}\text { Hamidreza Mohammadi } \\
\text { Iran }\end{array}$ \\
\hline $\begin{array}{l}\text { Iman Karimzadeh } \\
\text { Iran }\end{array}$ & $\begin{array}{l}\text { Mostafa Mohammadi } \\
\text { Iran }\end{array}$ \\
\hline $\begin{array}{l}\text { Mehdi Khabazkhoob } \\
\text { Iran }\end{array}$ & $\begin{array}{l}\text { Elham Mohit } \\
\text { Iran }\end{array}$ \\
\hline $\begin{array}{l}\text { Ali Khalaj } \\
\text { Iran }\end{array}$ & $\begin{array}{l}\text { Mojtaba Mojtahedzadeh } \\
\text { Iran }\end{array}$ \\
\hline $\begin{array}{l}\text { Hossein Khalili } \\
\text { Iran }\end{array}$ & $\begin{array}{l}\text { Aram Mokarizadeh } \\
\text { Iran }\end{array}$ \\
\hline $\begin{array}{l}\text { Mahnaz Khanavi } \\
\text { Iran }\end{array}$ & $\begin{array}{l}\text { Dariush Moslemi } \\
\text { Iran }\end{array}$ \\
\hline $\begin{array}{l}\text { Mohsen Khatibi } \\
\text { Iran }\end{array}$ & $\begin{array}{l}\text { Sara Mostafalou } \\
\text { Iran }\end{array}$ \\
\hline $\begin{array}{l}\text { Payam Khazaeli } \\
\text { Iran }\end{array}$ & $\begin{array}{l}\text { Amrollah Mostafazadeh } \\
\text { Iran }\end{array}$ \\
\hline $\begin{array}{l}\text { Reza Khodarahmi } \\
\text { Iran }\end{array}$ & $\begin{array}{l}\text { Guoying } \mathbf{M u} \\
\text { Chile }\end{array}$ \\
\hline $\begin{array}{l}\text { Mehdi Khoobi } \\
\text { Iran }\end{array}$ & $\begin{array}{l}\text { Hamid Nadri } \\
\text { Iran }\end{array}$ \\
\hline $\begin{array}{l}\text { Mehdi Khoshneviszadeh } \\
\text { Iran }\end{array}$ & $\begin{array}{l}\text { Nastaran Nafissi-Varcheh } \\
\text { Iran }\end{array}$ \\
\hline $\begin{array}{l}\text { Maija Konstenius } \\
\text { Sweden }\end{array}$ & $\begin{array}{l}\text { Chanin Nantasenamat } \\
\text { Thailand }\end{array}$ \\
\hline
\end{tabular}




\begin{tabular}{ll} 
Latifeh Navidpour & Amin Rostami-Hodjegan \\
Iran & United Kingdom \\
Shekoufeh Nikfar & Daniele Rubert Nogueira \\
Iran & Brazil \\
Ali Nokhodchi & Mina Saeedi \\
United Kingdom & Iran \\
Mohsen Pakzad & Soodabeh Saeidnia \\
Iran & Iran \\
Hadi Parsian & Maliheh Safavi \\
Iran & Iran \\
Poopak Pir & Mahmood Saghaei \\
Iran & Iran \\
Morteza Pirali-Hamedani & Amirhossein Sakhteman \\
Iran & Iran \\
Mahdi Pouramir & Pooneh Salari \\
Iran & Iran \\
Eslam Pourbasheer & Sajad Salehi \\
Iran & United States of America \\
Mohammad Reza Pourmand & Satyajit Sarker \\
Iran & United Kingdom \\
Atiar Rahman & Parisa Sarkhail \\
Bangladesh & Iran \\
Sara Rasoul-Amini & Habib A. Shojaei Saadi \\
Iran & Canada \\
Mohammad Amin Rezvanfar & Fatemeh Soleymani \\
Iran & Iran \\
& \\
\hline &
\end{tabular}

Kambiz Soltaninejad

Iran

Azita Hajhossein Talasaz

Iran

Nasser Vahdati-Mashhadian

Iran

Ronny Vargas

Costa Rica

Alireza Vatanara

Iran

Ana Vila Verde

Germany

Khushwant S. Yadav

India

Che-Ming Yang

Taiwan

Mohammad Hossein Yazdi

Iran

Fatemeh Yousefbeyk

Iran

Ebrahim Zabihi

Iran

Bijan Zare

Iran

Mohsen Zeeb

Iran 